\title{
AN INVARIANCE THEOREM FOR REPRESENTATIONS OF BANACH ALGEBRAS
}

\author{
L. TERRELL GARDNER ${ }^{1}$
}

1. Introduction. Under certain circumstances, a topological linear space acted upon by a given linear transformation $\tau$ can be shown to admit the action of a suitably continuous one-parameter group of transformations containing $\tau$, and associated with $\tau$ in a natural way. An illustration is afforded by the following theorem, which plays an important auxiliary role in [1]:

Let $\mathfrak{S}$ be a norm-closed linear subspace of $\mathfrak{R}(\mathfrak{H C})$. Then if $\mathcal{S}$ is invariant under $A \rightarrow T^{-1} A T, S$ is invariant also under $A \rightarrow A \log T-(\log T) A$, and under $A \rightarrow T^{-s} A T^{\text {s }}$ for all real numbers $s$.

(Here $\mathfrak{H C}$ is a Hilbert space, and $\mathfrak{R}(\mathfrak{F C})$ the algebra of all bounded linear operators on $\mathfrak{H C} . T$ is a positive, regular element of $\mathfrak{R}(\mathfrak{H C})$.)

It is the purpose of this note to provide an appropriately general setting for this theorem. Our main result is the:

INVARIANCE THEOREM. Let $\mathfrak{B}$ be a Banach algebra, $x$ and $y$ elements of $\mathfrak{B}$ with spectra in the open right half-plane $H^{+}$. Let $\phi$ be a strongly continuous representation of $\mathfrak{B}$ on the topological linear space $\mathcal{E}$, with $\phi(x y)=\phi(y x)$. Then every closed subspace $S$ of $\&$ invariant under $\phi(x y)$ is invariant also under $\phi(\log x+\log y)$ and under $\phi\left(x^{s} y^{s}\right)$ for all real numbers $s . s \rightarrow \phi\left(x^{s} y^{s}\right)$ is a strongly continuous one-parameter group in $\mathfrak{R}(\mathcal{S})$.

(Here $z^{a}=\exp (s \log z$ ), where log is defined for complex $\alpha$ in the complement $\Sigma$ of the nonpositive real numbers $R^{-}$by taking arg $\alpha$ $\in(-\pi, \pi)$, and then for $z$ in $\mathfrak{B}$ with spectrum $\sigma(z) \subset \Sigma$ by Cauchy's formula $[2, \S \S 5.2,5.4],[5$, Theorem 3.5.1].)

\section{Preliminary results.}

LEMma 1. Let $\mathfrak{B}$ be a Banach algebra with identity element, $x$ and $y$ commuting regular elements of $\mathfrak{B}$ with spectra in $H^{+}$. Then $\log (x y)$ $=\log x+\log y$.

Proof. We first remark that $\sigma(x y) \subset \sigma(x) \sigma(y)=\{r s \mid r \in \sigma(x)$, $s \in \sigma(y)\}$. In fact, let $\mathfrak{B}^{\prime}$ be a maximal commutative subalgebra of $\mathfrak{B}$

Received by the editors September 1, 1964.

${ }_{1}$ This work was supported in part by the National Science Foundation under Grant NSF GP-1604. 
containing $x$ and $y$. Then the injection map of $\mathfrak{B}^{\prime}$ into $\mathfrak{B}$ is spectrumpreserving [5, Theorem 1.6.9]. If $\alpha \in \sigma(x y)$, we can choose a homomorphism $h$ of $\mathfrak{B}^{\prime}$ on to the complex numbers with $\alpha=h(x y)=h(x) h(y)$ $\in \sigma(x) \sigma(y)$, proving the remark. (The author is indebted to Professor R. V. Kadison for the simplicity of the above proof.)

From the hypothesis and the remark above, we conclude that $\sigma(x y) \subset \Sigma$, so that $\log (x y)$ is defined. By standard properties of the functional calculus for commutative Banach algebras [5, Theorem 3.5.1] we have for each element $z$ of $\mathfrak{B}^{\prime}$ with $\sigma(z) \subset \Sigma$, and for each homomorphism $h$ of $\mathfrak{B}^{\prime}$ onto the complex numbers,

$$
h(\log z)=\log h(z) \text {. }
$$

Now $\log (h(x) h(y))=\log h(x)+\log h(y)$, since $|\arg h(x)|<\pi / 2$, $|\arg h(y)|<\pi / 2$, showing the imaginary part of the sum on the right to be less than $\pi$ in absolute value.

We set $q=\log (x y)-\log x-\log y$ and show by a trivial computation that $h(q)=0$, whence $q \in$ radical $\mathfrak{B}^{\prime}$. On the other hand, $\exp (q)=1$, so that by a result of Lorch $[3$, p. $421 ; 2$, Theorem 5.5 .5$], q$ is a finite linear combination of idempotents $j$, which we may take to be mutually orthogonal. But then, since the radical is an ideal, each $j$ is in the radical. Finally, an idempotent in the radical must be zero, and $q=0$, as claimed.

Mergelyan [4] has shown that if $E$ is a compact subset of the complex plane, each function $f$ continuous on $E$ and analytic at interior points of $E$ is uniformly approximable on $E$ by polynomial functions if and only if $E$ does not divide the plane. For $z$ in $B$ with $\sigma(z) \subset \Sigma$, we apply Mergelyan's theorem to the case $f=\log , E=E^{\prime}+V$, where $E^{\prime}$ $=\left\{\alpha|| \alpha \mid \leqq\|z\| \& \operatorname{dist}\left(\alpha, R^{-}\right) \geqq 2 / 3 \operatorname{dist}\left(\sigma(z), R^{-}\right)\right\}$and $V$ is the closed disc of radius $1 / 2 \operatorname{dist}\left(\sigma(z), R^{-}\right)$centered at 0 in the complex plane. Then if $\left\{p_{n}\right\}$ is a sequence of polynomials such that $p_{n} \rightarrow \log$ uniformly on $E, p_{n}(z) \rightarrow \log (z)$ by [2, Theorem 5.2.5]. Thus $\log z, s \log z$, and finally $\exp (s \log z)=z^{8}$ are approximable by polynomials in $z$, provided only that $\sigma(z) \subset \Sigma$. An immediate consequence is that if $w$ and $z$ are commuting elements of $B$ with spectra in $\Sigma$, then $\log w, \log z$, $w^{8}$ and $z^{z}$ lie in the closed commutative algebra generated by $w, z$ and the unit element $e$. Then, returning to the notation and hypotheses of Lemma 1 we have:

Corollary 1. $x^{s} y^{s}=(x y)^{s}$.

Proof. Clear.

Corollary 2. $x^{8} y^{8}$ is a limit of polynomials in $x y$.

This is immediate from Corollary 1 and the preceding remarks. 
3. Proof of the main result. The strong continuity of $\phi$ means that for each vector $v$ of $\mathcal{E}$, the mapping $x \rightarrow \phi(x)_{v}$ is continuous on $\mathfrak{B}$ to $\mathcal{E}$. Equivalently, $\phi$ is continuous on $\mathfrak{B}$ with its norm topology to $\mathfrak{R}(\mathcal{E})$ in the point-open topology. Thus the kernel $\mathfrak{M}$ of $\phi$ is a closed, twosided ideal in $\mathfrak{B}$, and $\phi$ defines a strongly continuous and faithful representation $\phi_{*}$ of $\mathfrak{B} / \mathfrak{M}$ on $\mathcal{E}$. Our proof is a straightforward reduction from $\mathfrak{B}$ to $\mathfrak{B} / \mathfrak{M}$.

The natural map $\mu$ of $\mathfrak{B}$ onto $\mathfrak{B} / \mathfrak{M}$ is continuous, and preserves polynomials, so that $\mu \circ \exp =\exp \circ \mu$. Moreover, since $\sigma\left(z_{*}\right) \subset \sigma(z)$ (for $z$ in $\mathfrak{B}, z_{*}=\mu(z)$ ), we may conclude that if $\sigma(z) \subset H^{+}(\operatorname{resp} . \Sigma)$, then $\sigma\left(z_{*}\right) \subset H^{+}($resp. $\Sigma)$, and in either case $\log z_{*}$ exists and $(\log z)_{*}$ $=\log \left(z_{*}\right),\left(z^{s}\right)_{*}=\left(z_{*}\right)^{s}$. Finally, $x_{*} y_{*}=y_{*} x_{*}$, because $\phi(x y)=\phi(y x)$ by hypothesis. Applying Corollary 2 (and its proof) to these commuting elements of $\mathfrak{B} / \mathfrak{M}$, we conclude that $x_{*}^{s} y_{*}^{s}$ and $\log \left(x_{*}^{s} y_{*}^{s}\right)$ are limits of polynomials in $x_{*} y_{*}$. But then if $S$ is a closed subspace of $\varepsilon$ invariant under $\phi(x y)=\phi_{*}\left(x_{*} y_{*}\right), s$ is invariant also under $\phi\left(x_{*}^{s} y_{*}^{s}\right)=\phi_{*}\left(x_{*}^{s} y_{*}^{s}\right)$ and under $\phi(\log x+\log y)=\phi_{*}\left(\log x_{*}+\log y_{*}\right)=\phi_{*}\left(\log \left(x_{*} y_{*}\right)\right)$, which was to be shown.

That $s \rightarrow \phi\left(x^{8} y^{8}\right)$ is a strongly continuous group of endomorphisms of $S$ is clear from the above considerations.

4. Application to inner automorphisms. In this section, we recover the first-cited theorem of the introduction, in considerably greater generality.

Corollary 3. Let $\mathfrak{A}$ be a Banach algebra with unit element, $z$ an element of $\mathfrak{A}$ with $\sigma(z) \subset H^{+}$. Let $\mathfrak{S}$ be a closed subspace of $\mathfrak{A}$ invariant under $x \rightarrow z^{-1} x z$. Then $S$ is invariant under $x \rightarrow x \log z-(\log z) x$ and under $x \rightarrow z^{-8} x z^{8}$ for all real numbers $s$.

Proof. We know that $z^{-1}$ exists, and that $\sigma\left(z^{-1}\right) \subset H^{+}$, also. In the statement of the theorem, take $\mathfrak{B}=\mathfrak{R}(\mathfrak{A}), \mathcal{E}=\mathfrak{A}$, and $\phi=$ identity. Denote by $w \otimes I(I \otimes w)$ the transformation $v \rightarrow w v(v \rightarrow v w)$ for $w$ in $\mathfrak{A}$, and set $x=z^{-1} \otimes I, y=I \otimes z$. Recalling that the left- and right-regular representations of a Banach algebra $\mathfrak{A}$ with identity are spectrumpreserving isometric isomorphisms of $\mathfrak{A}$ onto mutually commuting subalgebras of $\mathfrak{I}(\mathfrak{H})$, [5, Chapter 1$]$ we find the hypotheses of the theorem verified in the above interpretation. We conclude from the theorem that $S$ is invariant under $\log \left(z^{-1} \otimes I\right)+\log (I \otimes z)$ and under $\left(z^{-1} \otimes I\right)^{s}(I \otimes z)^{s}$ for all real $s$. But these are $I \otimes \log z-\log z \otimes I$ and $z^{-s} \otimes z^{s}$, respectively. In fact, polynomial approximations via Mergelyan's theorem, together with the remark above on the regular representations, afford immediate verification. 


\section{BIBLIOGRAPHY}

1. L. T Gardner, On isomorphisms of $C^{*}$-algebras, Amer. J. Math. 87 (1965), 384-396.

2. E. Hille and R. S. Phillips, Functional analysis and semi-groups, Amer. Math. Soc. Colloq. Publ. Vol. 30, Amer. Math. Soc., Providence, R. I., 1957.

3. E. R. Lorch, The theory of analytic functions in normed abelian vector rings, Trans. Amer. Math. Soc. 54 (1943), 414-425.

4. S. N. Mergelyan, On the representation of functions by series of polynomials, Dokl. Akad. Nauk SSSR 78 (1951), 405-408; English Transl., Amer. Math. Soc. Transl. No. 85, Amer. Math. Soc., Providence, R. I., 1953.

5. C. E. Rickart, General theory of Banach algebras, Van Nostrand, New York, 1960.

Columbia University 